\title{
Study on Huge Type of Invasive Pituitary Adenoma through Frontotemporal Orbital Zygomatic into The Applied Anatomy Study of the Road
}

\author{
Cailian Ruan ${ }^{1}$, Tao Xue ${ }^{2}$, Yuanyuan Xie $^{3}$ \\ ${ }^{1}$ School of Medicine, Yan'an University, Yan'an, Shaanxi, 716000 \\ ${ }^{2}$ Affiliated Hospital of Yan'an University, Yan'an, Shaanxi, 716000 \\ 346591653@163.com
}

KEYWORDS: Huge Type of Invasive Pituitary Adenomas; Frontotemporal Orbital Zygomatic Approach; Anatomical Study

\begin{abstract}
To explore the type giant invasive pituitary adenomas by frontotemporal orbital zygomatic into the applied anatomy study of the road.Methods: 15 cases of pituitary adenomas in frontotemporal orbital zygomatic into the anatomy of the application of the road.Put the 15 cases of skull in it for fixed and Ma Linzhong side of 30 samples, adopts the frontotemporal orbital zygomatic approach surgery for each specimen microdissection. By adopting the retrospective summary of frontotemporal microdissection orbital zygomatic type into the road treatment in giant invasive pituitary adenomas in the anatomical study 5 patients of our hospital is for surgery. Results: through the specimen of microdissection combining retrospective data, you can see that in the stage of epidural want from multiple clearance to know pituitary adenoma resection of the tumor needs growth direction.Resection of giant invasive pituitary adenomas should be combined with epidural cavernous wall into the road, in order to achieve the goal of excision.In 5 patients, all 3 cases, all cut in 1 case, partial resection in 1 case., according to the conclusion: the surgery for patients with invasive pituitary adenomas huge watch can use frontotemporal orbital zygomatic approach surgery for targeted treatment, and can completely proper cutting according to patients condition, to the health of the patients to provide more opportunities.
\end{abstract}

\section{Introduction}

Processing giant invasive pituitary adenomas disease with current medical development is still more difficult, more complicated surgery, and surgery can not be total removal of the tumor, the virus will develop the middle cranial fossa were cavernous sinus invasion and the King intra-arterial invasion sphenoid sinus. There are a variety of pituitary adenomas, in general, divided into craniotomy and transsphenoidal approach, no matter what kind of surgery can not be a one-time removal of pituitary adenoma, and the virus will spread outward, affecting the cavernous sinus , sphenoid sinus and middle cranial fossa and other parts. If such a situation occurs it becomes more complicated. Specific conditions reported below.

\section{Materials and Methods}

\section{A. Anatomic and Clinical Data Material}

Formalin and 30 specimens were normal and did not have signs of trauma and intracranial normal. Use dedicated stainless steel head frame, burr, special equipment craniotomy and microsurgical instruments. 
To our hospital five cases of giant invasive pituitary adenoma patients with frontotemporal orbital zygomatic surgery treatment, 2 males and 3 females, aged 15 --- 72 years. All patients underwent MRI scans. 5 patients with tumor diameter in $4-5 \mathrm{~cm}$ of one case and are more than four cases of $5 \mathrm{~cm}$.

\section{B. Methods}

After the outside of the cavernous sinus surgery, the fossa dura proper release to the hole, then from the outer jaw nerve meninges cut into the dura dissection, the proper separation of the trigeminal nerve to the surrounding, the goal is to separate the orbital crack on top, top and cavernous sinus lateral wall of the cavernous sinus and so on. In order for the safety of the operation there is a good control requires internal carotid artery rock unit fully exposed, can effectively control blood flow to reduce the risk of surgery. Inside of the front of the cavernous sinus approach to ACP appropriately abrade clinoid structure will be able to varying degrees of exposure, and open walls and optic nerve sheath, cut the sidewall dura within CS, and cut the saddle diaphragm, so the aim is to clinoid outwardly dura retractor. After the outside before the inside of the retractor have a cavernous space, through this gap can be observed for the entire pituitary fossa, the purpose of tumor resection. After removal of the orbital apex ophthalmic artery, between the IC and the optic nerve segment gap period for operation, the sudden removal of the bed and open the optic canal wall, the length of the optic nerve can be amplified, and can increase the width of optic carotid triangle. Much of this can be improved clinoid around the operative field.

\section{Results}

15 patients with pituitary adenoma were dissected application frontotemporal orbital zygomatic road. The 15 cases of head into formalin fixed and collected 30 specimens, using frontotemporal orbital zygomatic approach to surgical microdissected for each specimen. Method A is the summary the frontotemporal orbital zygomatic approach for treatment of anatomical studies of giant invasive pituitary adenomas according to microdissection and 5 patients in our hospital for surgery. The results showed that five cases of giant invasive pituitary adenoma patients with total resection in 3 cases, subtotal of 1 case, 1 case of partial resection. 5 patients after treatment of the disease are to a certain control, not the number of deaths. They have suffered different degrees of complications, 3 cases of diabetes insipidus, electrolyte imbalance 2 cases, 1 case of temperature offset by targeted treatment to completely cure the symptoms of electrolyte imbalance and temperature offset, three cases of diabetes insipidus 2 patients returned to normal within two weeks, one case returned to normal within three months.

\section{Discussion}

Full-time cut giant invasive pituitary adenomas by dissecting through complex experimental study surgical anatomy concluded that frontotemporal craniotomy method can orbitozygomatic the maximum extent of tumor resection in different locations at once, by the sponge medial sinus surgery through the cavernous sinus surgical anatomy and traditions are very different, dealing mainly with surgery medial triangular shaped chiasm pituitary adenoma, cut away ICA ring, near the ring, saddle compartment and other protruding section, between the use of the gap of the cavernous sinus lesions appropriately removed. However, under certain circumstances, because the dura is not complete, it is prone to venous plexus bleeding, so this method of operation can not be totally removed the tumor. Before the medial cavernous surgery has several advantages: 1) the procedure of the operation is relatively simple and safe, and the surgical field is more open; 2) in 
the course of the operation can not respond to each ring of the dura mater is opened, so it there would not be the case cavernous venous plexus bleeding, there is great security; range 3) surgical procedures more widely, can handle a variety of locations lesions, such as knot epidural approach capable of saddle, under saddle and lesions on both sides for processing.

Resection of the tumor has a variety of approaches, but the most effective and the most important part is to account for transcranial surgical approach in resection, because they have more pituitary tumor resection need to adopt this approach path removed. If there is pituitary tumor resection path need to use this method of approach must be taken to this method, which also need to tumor growth and direction of a great extent. In our clinical treatment, the use of such surgical approach is still relatively more, so the need to standardize the default time for such surgery. In order for the safety of the operation there is a good control requires internal carotid artery rock unit fully exposed, can effectively control blood flow to reduce the risk of surgery. Inside of the front of the cavernous sinus approach to ACP appropriately abrade clinoid structure will be able to varying degrees of exposure, and open walls and optic nerve sheath, cut the sidewall dura within CS, and cut the saddle diaphragm, so the aim is to clinoid outwardly dura retractor. After the outside before the inside of the retractor have a cavernous space, through this gap can be observed for the entire pituitary fossa, the purpose of tumor resection. After removal of the orbital apex ophthalmic artery, between the IC and the optic nerve segment gap period for operation, the sudden removal of the bed and open the optic canal wall, the length of the optic nerve can be amplified, and can increase the width of optic carotid triangle. Much of this can be improved clinoid around the operative field.

After this kind of surgery for tumor resection path need to pay attention to some aspects, if you approach from the outside of the dura was resected adenoma cavernous sinus tumor is more rigid, when radical resection can not be the purpose of removal, because this would artery within the cavernous sinus or great trauma to the brain. After excision of the tumor within the cavernous sinus, venous bleeding is likely to occur, under normal circumstances the use of a sponge with thrombin to control. Subdural approach when surgery requires careful not damage the optic nerve and cross arteries. Under normal circumstances is benign pituitary adenoma, not coated, but in the specific operation of the capsule, the thickness of this envelope is thinner, it belongs to the pituitary gland, the pituitary stalk and other envelope, for example, tumor capsule and saddle diaphragm interlinkages between the two is very thin envelope and, if the end of the saddle by a certain degree of damage is required in the pituitary fossa muscle padding.

For huge tumors of different treatment methods are different, the patient's condition before surgery for close observation and analysis, to develop a proper surgical plan and expected treatment goals, the choice of surgical methods are mainly the following aspects: 1) First, You should look at the type of tumor, and the extent of the size and morphology of tumors invasion; 2) the direction of tumor growth also need to have a more clear understanding; 3) understand the hormonal status; 4) must be taken into surgery brought all aspects of risk. In many cases it can not be good for surgical diffuse invasive adenoma treatment, because the human dura mater and brain tumors were invasive, so, with the best surgical method can not resection. The maximum effect of surgery is to reduce to the maximum extent under the premise of the complications of tumor resection. Ready for the next treatment. Our country frontotemporal orbital zygomatic surgical methods in clinical remains a more important role, so, neurosurgeons should maintain the safety and effectiveness of this approach technology.

The results showed that five cases of giant invasive pituitary adenoma patients with total resection in 3 cases, subtotal of 1 case, 1 case of partial resection. They have suffered different degrees of complications, 3 cases of diabetes insipidus, electrolyte imbalance 2 cases, 1 case of 
temperature offset by targeted treatment to completely cure the symptoms of electrolyte imbalance and temperature offset, three cases of diabetes insipidus 2 patients returned to normal within two weeks, one case returned to normal within three months. Dural stage, want to cut the tumor from the multi-gap need to know the direction of the pituitary adenoma growth. Of giant invasive pituitary adenoma excision should be combined with epidural sponge outer wall approach, in order to achieve the purpose of removal. In 5 patients, the total resection in 3 cases, subtotal in 1 case, partial resection in 1 case. By a surgical data show that for patients with giant invasive pituitary adenomas can be used to see frontotemporal orbital zygomatic approach targeted surgical treatment, and can be appropriately cropped depending on the patient's condition.

\section{REFERENCES}

[1] Tao Cunshan. Wu Yongkang. Cheng Cheng,etc. US Microanatomy and clinical application of transcranial pituitary adenoma surgery [J]. anatomical study, 2014,11 (05): 331-334.

[2] Ren Yuan. Invasive pituitary adenomas through improved diagnosis and pterional microsurgical treatment [D]. Central South University, 2009.11.

[3] Zhang Rongwei. Expanding Transsphenoidal treatment cavernous invasive pituitary adenomas Study [D]. Third Military Medical University, 2007,5.

[4] Wang Renzhi, Yin Jian. Expanded transsphenoidal removal of invasive pituitary adenomas [J]. Journal of Surgery, 2014,22 (65): 1548-1550.

[5] SuYong yong, Bai Kai, Yuan Shaoji, etc. Pterional microsurgical treatment of invasive pituitary adenomas [J]. Jilin Medicine, 2010,27: 4663-4665.

[6] Tao Cunshan. Expression of nerve growth, the United States House Qing Ji Chang transcranial removal of giant invasive pituitary adenomas microsurgery anatomy [J]. Jiangsu Medical Journal, 2013,25 (05): 355-357.

[7] Chen Xiaoling. pterion postoperative care invasive pituitary adenomas [J]. Journal of Nursing Science, 2009,16 (33): 40-42.

[8] Du Yiran. Surgical treatment of pituitary adenomas [D]. Tianjin Medical University, 2013.

[9] Ye Jinping, Wang Baoping, Qin Li. through pterional microsurgical removal of invasive pituitary adenoma 18 cases [J]. Zhejiang Medicine, 1999,63 (03): 44-46.

[10] Huang Dejun, Xia Yucheng etc. pituitary adenoma surgical approach to select [J]. Ningxia Medical Journal, 2012,61 (04): 201-203.

[11] Chen title city, Li Shiqi. giant invasive pituitary adenoma 67 cases [J]. Chinese Journal of Nervous and Mental Diseases, 2013,01 (11): 64-65. 